\title{
Cepheids in the Nearby Galaxy IC 1613
}

\author{
E. Antonello, L. Mantegazza, D. Fugazza, M. Bossi, S. Covino \\ Osservatorio Astronomico di Brera, Via E. Bianchi 46, 23807 Merate, \\ Italy
}

\begin{abstract}
A summary of the first results of a search for Cepheids in IC 1613 is reported along with a short discussion of the adopted technique, a comparison of the characteristics of Cepheid light curves in the Galaxy, Magellanic Clouds and IC 1613, and a possible application for a $P-L$ relation derivation. First overtone Cepheids have been identified for the first time in a galaxy farther than the Magellanic Clouds.
\end{abstract}

\section{Introduction}

IC 1613 is a dwarf irregular, metal-poor galaxy in the Local Group, with a distance modulus of 24.42, similar to that of M31 (24.44). The Cepheids in this galaxy were studied by Sandage (1971) and Carlson \& Sandage (1990) on the basis of photographic data; Freedman (1988) made complementary CCD observations of the known Cepheids. Our project was to make a survey with CCD photometry for discovering new Cepheids and obtaining accurate light curves (Antonello et al. 1999a).

\section{Technique}

The observations were made during 1995-1998 at ESO La Silla with the Dutch 0.91-m telescope. Four fields of $3.8 \times 3.8$ arcmin were surveyed. Owing to the small size of the telescope, we decided to observe mainly without Any filter (white light or $W h$-band) to get the best photon statistics for variability studies; from a few $V$ and $R$ images we also got information on the color of the brighter stars. The advantage of the $W h$-band is that the number of collected photons is about five times that in the $V$-band for the same exposure time. The overall transmission of the optical system with a back-illuminated CCD detector is shown in Figure 1. Note that for a G-type star the effective wavelength of the photometric bands is 548,676 and $601 \mathrm{~nm}$ for $V, R$ and $W h$, respectively; that is, for Cepheids the effective wavelength of $W h$ is intermediate between that of $V$ and $R$. Of course, the $W h$-band is very wide, and the resulting photometry is significantly dependent on the instrumentation (e.g. front-illuminated or backilluminated CCD detector). However, for a given instrumentation it is possible to verify that $W h$ correlates well with $V$ and $V-R$, and in our case there is a relation of the type $V-W h=0.33(V-R)+0.51(V-R)^{2}$ between colors.

The advantage of $W h$ observations is shown also by the number of detected stars. $W h, V$ and $R$ images were taken during the same night with the same 


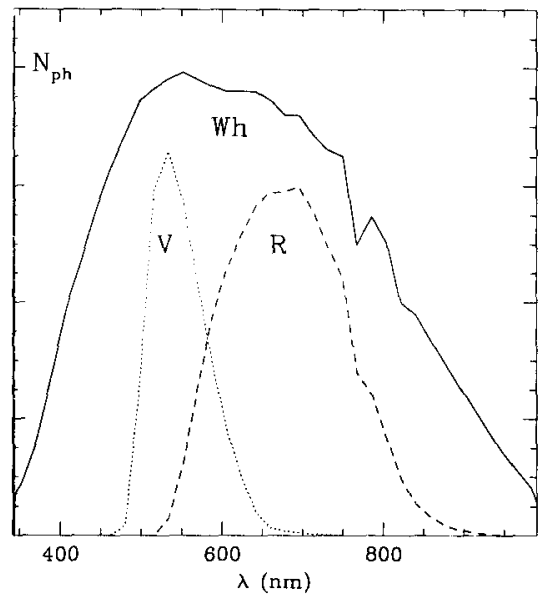

Figure 1. Number of photons (arbitrary scale) against wavelength for a $\mathrm{G}$-type star observed in $V, R$ and $W h$ bands

seeing conditions. The number of stars observed in the three bands is 1217,687 and 827 for $W h, V$ and $R$, respectively; from a comparison with Freedman's (1988) data it is possible to conclude that the color of stars observed in Wh, but not in $V$, band is typical of $\mathrm{F}-\mathrm{K}$ spectral type stars.

\section{Variable Stars}

For the present, 67 Wh images of one field (Field A) have been reduced using DAOPHOT (Stetson 1987). The data analysis has allowed us to identify 43 population I Cepheids, while only 9 were previously known in this field; most of the newly discovered stars have short periods. Those with known $V-R$ are located in the instability strip in agreement with the previous results of Freedman (1988). The luminosities, periods and relatively small amplitudes of a group of at least 8 Cepheids indicate that they are first overtone pulsators; the Fourier decomposition of the light curves confirms this characteristics. It is remarkable that the first detection of a group of first overtone Cepheids in a galaxy which is as far as M31 has been obtained with a 0.9-m telescope. No double-mode Cepheid has been found, probably because the precision and sampling of the data are not sufficient. Furthermore, at least 5 population II Cepheids and 8 eclipsing binaries have been observed; about 40 stars are probable long period, semiregular or irregular variables.

\section{Cepheid Light Curves in Different Galaxies}

The metallicity of IC 1613 is $[\mathrm{Fe} / \mathrm{H}] \sim-1.3$ (or $Z \sim 0.001$ ), to be compared with that of Galaxy $(\sim 0.0), \mathrm{LMC}(\sim-0.2)$, and SMC $(\sim-0.5)$. Therefore, it is an interesting case for an extension of the comparison of Fourier parameters of 

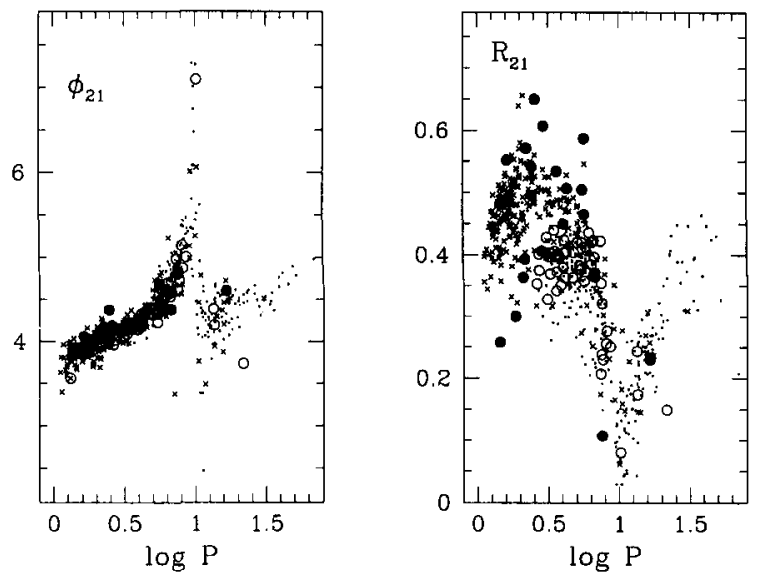

Figure 2. Phase difference $\phi_{21}$ and amplitude ratio $R_{21}$ against period for Cepheids in the Galaxy (dots), LMC (open circles), SMC (crosses) and IC 1613 (filled circles)

Cepheid light curves in various galaxies as done by Beaulieu \& Sasselov (1997), looking for metallicity effects. The comparison must be made bearing in mind the possible systematic differences given by the different passbands used for the observations (Antonello et al. 1999b). As an example, we show in Fig. 2 a detail of the diagrams of the phase difference $\phi_{21}$ and amplitude ratio $R_{21}$ against period $P$ for the fundamental mode Cepheids. The trend in the $\phi_{21}-P$ diagram appears to be the same for all the Cepheids in the four galaxies, while the largest $R_{21}$ values of shorter-period Cepheids appear to be dependent on the metallicity.

\section{5. $P-L$ Relation}

The Cepheid luminosity in $W h$ band is correlated with $P$, and one could obtain a $P-L$ relation using this band. However, it is possible to obtain a $P-L$ relation using standard photometry data by adopting the single-phase method devised by Freedman (1988); that is, with the only available $V$ observation of the (brightest) Cepheids and the characteristics of $W h$ light curves. Here we summarize the discussion reported by Antonello et al. (1999b). Cepheid light curve shapes and amplitudes depend on the color or passband; we take into account the ratio $\sim 0.8$ between $V$ and $W h$ amplitudes and for the present we shall ignore the small differences between $W h$ and $V$ light curve shape. The mean $V$ magnitude of Cepheids is estimated by the formula $V_{0}=V(\phi)-\left[W h(\phi)-W h_{0}\right] / 0.8$, where $V(\phi)$ is the single measurement and $W h(\phi)$ the value of the fitted $W h$ curve for the same phase. Actually, $V_{0}$ is a single-phase $V$ value converted to the mean value. Fig. 3 shows the $P-L$ diagrams for the $W h_{0}$-band observations (mean light curve value) and for those converted to the mean value $V_{0}$. In the latter case, the scatter of fundamental mode Cepheids around the $P-L$ relation 


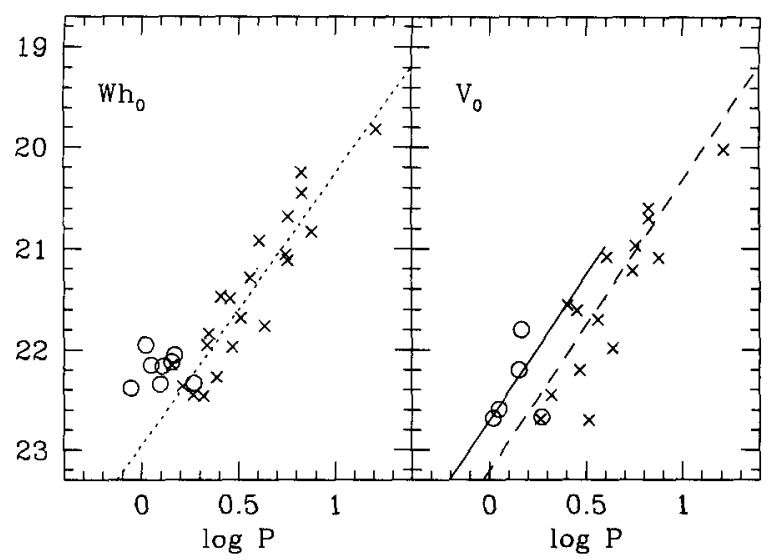

Figure 3. $\quad P-L$ diagrams for mean $W h$ (left panel) and converted to mean $V$ values (right panel). Crosses: fundamental mode Cepheids; open circles: first overtone mode Cepheids; dotted line (left panel): statistical relation for fundamental mode Cepheids; dashed line (right panel): standard $P-L$ relation (Freedman 1988) for fundamental mode Cepheids; continuous line: approximate relation for first overtone mode Cepheids

(Freedman 1988) increases with $V_{0}$ owing to the increasing uncertainty in the single $V$ measurement.

\section{References}

Antonello, E., Mantegazza, L., Fugazza, D., Bossi, M., \& Covino, S. 1999a, A\&A, in press, [astro-ph/9906483]

Antonello, E., Mantegazza, L., Fugazza, D., \& Bossi, M. 1999b, A\&A, submitted Beaulieu, J. P. \& Sasselov D. D. 1997, in Variable Stars and the Astrophysical Returns of Microlensing Surveys, ed. R. Ferlet, J. P. Maillard, \& B. Raban (Gif-sur-Yvette: Editions Frontières), 193

Carlson, G. \& Sandage, A. 1990, ApJ, 352, 587

Freedman, W. L. 1988, ApJ, 326, 691

Sandage, A. 1971, ApJ, 166, 13

Stetson, P. B. 1987, PASP, 99, 191

\section{Discussion}

Siobahn Morgan: Do you see metallicity $-\phi_{31}$ relations in your data that can be parameterized in a manner similar to the RR Lyrae $Z-\phi_{31}$ relation?

Elio Antonello: For the present we have not considered higher-order Fourier parameters. Yours is a good suggestion. 